\title{
Renoprotective and antioxidant effects of cilnidipine in hypertensive patients
}

\begin{abstract}
Takeshi Soeki $^{1,5}$, Mitsuhiro Kitani ${ }^{2,5}$, Kenya Kusunose ${ }^{1}$, Shusuke Yagi ${ }^{1}$, Yoshio Taketani ${ }^{1}$, Kunihiko Koshiba ${ }^{1}$, Tetsuzo Wakatsuki ${ }^{1}$, Shunsuke Orino ${ }^{3}$, Kazuhiro Kawano ${ }^{4}$ and Masataka Sata ${ }^{1}$

Cilnidipine, an L/N-type calcium channel blocker (CCB), has been reported to have more beneficial effects on proteinuria progression in hypertensive patients than amlodipine, an L-type CCB. The $\mathrm{N}$-type calcium channel blockade that inhibits renal sympathetic nerve activity might reduce glomerular hypertension by facilitating vasodilation of the efferent arterioles. However, the precise mechanism of the renoprotective effect of cilnidipine remains unknown. Because cilnidipine exerted significantly higher antioxidant activity than amlodipine in cultured human mesangial cells, we hypothesized that cilnidipine might exert a renoprotective effect by suppressing oxidative stress. A total of 35 hypertensive patients receiving a renin-angiotensin system inhibitor were randomly assigned to a cilnidipine $(n=18 ; 10 \mathrm{mg}$ per day cilnidipine titrated to $20 \mathrm{mg}$ per day) or amlodipine ( $n=17 ; 5 \mathrm{mg}$ per day amlodipine titrated to $10 \mathrm{mg}$ per day) group; the target blood pressure (BP) was set at $130 / 85 \mathrm{mmHg}$. After 6 months of treatment, systolic and diastolic BPs were significantly reduced in both of the groups, without any significant difference between the groups. The urinary albumin, 8-hydroxy-2'-deoxyguanosine (OHdG) and liver-type fatty-acid-binding protein (L-FABP) to creatinine ratios significantly decreased in the cilnidipine group $(P<0.05)$ compared with those in the amlodipine group. The reductions in urinary albumin, 8-OHdG and L-FABP were not correlated with the change in systolic BP. In conclusion, cilnidipine, but not amlodipine, ameliorated urinary albumin excretion and decreased urinary 8-OHdG and L-FABP in the hypertensive patients. Cilnidipine probably exerts a greater renoprotective effect through its antioxidative properties.
\end{abstract}

Hypertension Research (2012) 35, 1058-1062; doi:10.1038/hr.2012.96; published online 5 July 2012

Keywords: amlodipine; antioxidative property; cilnidipine; renoprotective effect

\section{INTRODUCTION}

Patients with chronic kidney disease (CKD) are at a high risk of cardiovascular disease. ${ }^{1}$ Angiotensin-converting enzyme inhibitors (ACEIs) and angiotensin II type 1 receptor blockers (ARBs) are beneficial in suppressing the progression of $\mathrm{CKD}^{2,3}$ and are generally used as first-line treatments in hypertensive patients with CKD. Calcium channel blockers (CCBs) are known to have potent antihypertensive effects and are considered the main candidates in combination treatment with ACEIs or ARBs in hypertensive patients with CKD.

However, evidence of the renoprotective effect of various types of CCBs is inconsistent. This inconsistency might be due to the different specificity of each CCB for different calcium channels. Although cilnidipine, an L/N-type CCB, could suppress renal injury in hypertensive patients, ${ }^{4,5}$ amlodipine, an L-type CCB, could not. ${ }^{3,6}$ Furthermore, cilnidipine was shown to be more beneficial than amlodipine in combined treatment for hypertensive patients with kidney disease and significant proteinuria who were receiving treatment with a renin-angiotensin system (RAS) inhibitor. ${ }^{7}$ The beneficial effect of cilnidipine might be attributable to the inhibition of renal sympathetic nerve activity ${ }^{8}$ and reduction of glomerular hypertension (because of the vasodilation of efferent arterioles), which are consequences of the N-type calcium channel blockade. However, the precise mechanism underlying the renoprotective effect of cilnidipine remains unknown. Because cilnidipine, which has marked lipophilicity, ${ }^{9}$ showed significantly higher antioxidant activity in cultured human mesangial cells than did amlodipine (15), we hypothesized that cilnidipine might exert its renoprotective effects by suppressing oxidative stress.

Extensive experimental evidence suggests that lipids in the cellular membranes, as well as protein and DNA, are exposed to oxidative damage. In nuclear and mitochondrial DNA,

${ }^{1}$ Department of Cardiovascular Medicine, Institute of Health Biosciences, University of Tokushima Graduate School, Tokushima, Japan; ${ }^{2}$ Department of Cardiovascular Medicine, Kagawa Prefectual Shirotori Hospital, Higashikagawa, Japan; ${ }^{3}$ Department of Cardiovascular Medicine, Tokushima City Hospital, Tokushima, Japan and ${ }^{4}$ Department of Cardiovascular Medicine, Oe Kyodo Hospital, Yoshinogawa, Japan

${ }^{5}$ These two authors contributed equally to this work.

Correspondence: Dr T Soeki, Department of Cardiovascular Medicine, Institute of Health Biosciences, University of Tokushima Graduate School, 2-50-1 Kuramoto, Tokushima 770-8503, Japan.

E-mail: soeki@clin.med.tokushima-u.ac.jp

Received 27 February 2012; revised 3 April 2012; accepted 21 April 2012; published online 5 July 2012 
8-hydroxy-2'-deoxyguanosine $(\mathrm{OHdG})$ is one of the predominant sources of free radical-induced oxidative stress. ${ }^{10}$ Therefore, 8 -OHdG has widely been used as a biomarker of oxidative stress and has been shown to be associated with the progression of CKD. ${ }^{11,12}$ Urinary liver-type fatty-acid-binding protein (L-FABP) is a useful biomarker for renal tubulointerstitial injury and can be used to monitor both hemodynamic and drug responses in animal models and CKD patients. ${ }^{13,14}$ In addition, urinary L-FABP is more sensitive than urinary protein in predicting the progression of CKD. ${ }^{13}$

In the present study, the urinary excretion levels of $8-\mathrm{OHdG}$ and L-FABP were used as markers of oxidative stress and tubular injury, respectively. In addition, the antioxidative and antiproteinuric effects of cilnidipine were compared with those of amlodipine in hypertensive patients who were receiving treatment with a RAS inhibitor.

\section{METHODS}

\section{Study design}

In this 6-month-long, multi-center, prospective, randomized, open-labeled clinical trial named Tokushima Antioxidation Clinical Trial in Hypertensives, Cilnidipine vs. Amlodipine (TACTICAL) study, 35 hypertensive patients receiving RAS treatment (ACEI or ARB) at four hospitals in Japan were included between March 2009 and January 2011. The study was approved by the Institutional Review Board of The University of Tokushima Clinical Research Center and the review board of the four hospitals. The study protocol was in accordance with the Declaration of Helsinki Principles. Written informed consent was obtained from all the patients included in this study. The enrollment criteria were: (1) hypertension defined as systolic and diastolic blood pressure (BP) of $\geqslant 140 / 90 \mathrm{mmHg}$, (2) treatment with a RAS inhibitor for 3 months or more before the administration of cilnidipine or amlodipine and (3) serum creatinine level of $\leqslant 2.0 \mathrm{mg} \mathrm{dl}^{-1}$. The exclusion criteria were as follows: (1) age $<45$ years or $>85$ years, (2) hypertensive emergency, (3) severe heart failure, severe arrhythmia, angina, myocardial infarction and stroke within 6 months of the start of the trial, (4) pregnancy and (5) history of severe adverse effects of CCBs and RAS inhibitors.

The overview of the study design is shown in Figure 1. The subjects were randomly assigned to two groups at the time of registration. The patients received either of the following treatment regimens: $10 \mathrm{mg}$ per day cilnidipine, which was titrated up to daily doses of $20 \mathrm{mg}$, or $5 \mathrm{mg}$ per day amlodipine, which was titrated up to daily doses of $10 \mathrm{mg}$, to achieve the BP-target levels.

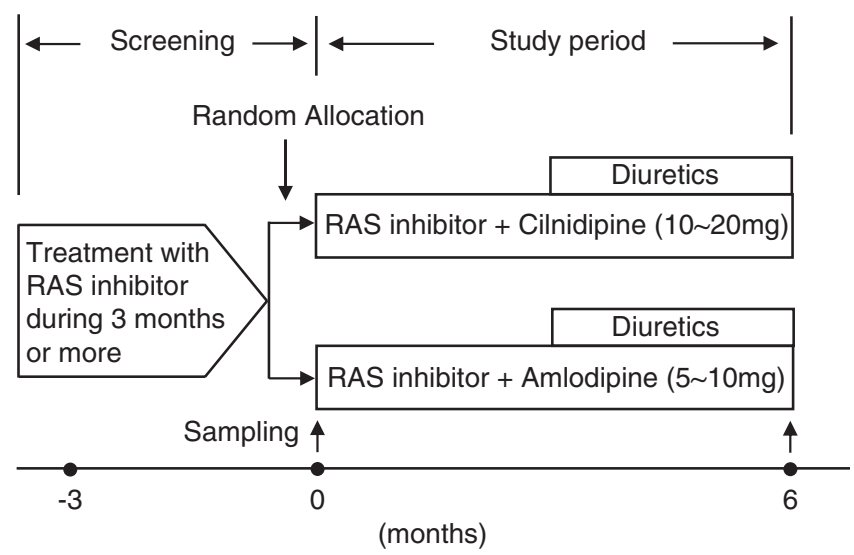

Figure 1 Study design. The patients received either of the following treatment regimens: $10 \mathrm{mg}$ per day cilnidipine, which was increased to daily doses of $20 \mathrm{mg}$, and $5 \mathrm{mg}$ per day amlodipine, which was increased to daily doses of $10 \mathrm{mg}$. The dose of the RAS inhibitors was not altered during the study period. The target BP level was $130 / 85 \mathrm{mmHg}$. If cilnidipine or amlodipine in combination with the RAS inhibitor failed to reduce the BP to the target level within 3 months, additional antihypertensive medications, such as diuretics, were administered to achieve the target BP.
The doses of ARB and ACEI were not altered during the study period. The target BP level was 130/85 mmHg. BP was measured according to the Japanese Society of Hypertension Guidelines for Measurement of Hypertension. ${ }^{15}$ During the first 3 months of the study, the patients were administered the assigned combination drug regimen at baseline. However, if cilnidipine or amlodipine in combination with the RAS inhibitor failed to reduce the BP to the target level within 3 months, additional antihypertensive medications, such as diuretics, were administered to achieve the target BP.

Dyslipidemia was defined according to the criteria established by the Japan Atherosclerosis Society (low-density lipoprotein $\geqslant 140 \mathrm{mg} \mathrm{dl}^{-1}$, trigylcerides $\geqslant 150 \mathrm{mg} \mathrm{dl}^{-1}$ and/or high-density lipoprotein $\left.<40 \mathrm{mg} \mathrm{dl}^{-1}\right)^{16}$ in addition to a medical history of hypercholesterolemia.

\section{Measurements of urinary parameters}

Morning-spot urine samples were obtained. The urinary levels of albumin and creatinine were measured using turbidimetric immunoassay and enzyme assay, respectively (Mitsubishi Chemical Medience, Tokyo, Japan). The urinary levels of 8-OHdG and L-FABP were measured using the sandwich enzyme-linked immunosorbent assay kit (NOF, Tokyo, Japan, and Immuno-Biological Lab, Tokyo, Japan). Urinary albumin, 8-OHdG and L-FABP levels were expressed as the ratio to the urinary creatinine concentration.

\section{Statistical analysis}

Data are expressed as the mean \pm s.d. Differences between the two groups were analyzed by Student's unpaired $t$-test for continuous variables and Chi-square test for categorical variables. The changes between before and after the administration of the therapy were assessed by Wilcoxon's signed-rank test. Correlations between two parameters were assessed using simple linear regression.

\section{RESULTS}

\section{Baseline characteristics}

No adverse effects related to the antihypertensive treatment occurred during the study period. The baseline characteristics of the subjects are shown in Table 1. There were no significant differences in age, gender, systolic and diastolic BPs, body mass index, diabetes mellitus incidence and dyslipidemia between the two groups. There were also no significant differences in the use of ARB/ACEI, beta-blockers and statins between the two groups.

\section{Changes in $\mathrm{BP}$ and heart rate}

Systolic and diastolic BPs were significantly lower in both the cilnidipine and amlodipine groups at 6 months after the

Table 1 Baseline characteristics

\begin{tabular}{|c|c|c|c|}
\hline & Cilnidipine group & Amlodipine group & P-value \\
\hline$n$ & 18 & 17 & \\
\hline Age (years) & $67.0 \pm 9.8$ & $68.6 \pm 8.7$ & NS \\
\hline Sex (male/female) & $12 / 6$ & $11 / 6$ & NS \\
\hline Systolic BP (mmHg) & $154.0 \pm 12.5$ & $154.6 \pm 13.3$ & NS \\
\hline Diastolic BP (mmHg) & $87.2 \pm 12.8$ & $89.3 \pm 9.9$ & NS \\
\hline Heart rate (bpm) & $69.3 \pm 9.3$ & $70.5 \pm 5.4$ & NS \\
\hline Body mass index $\left(\mathrm{kg} \mathrm{m}^{-2}\right)$ & $23.9 \pm 4.3$ & $23.7 \pm 3.9$ & NS \\
\hline Diabetes mellitus $(n)$ & $3(17 \%)$ & $4(23 \%)$ & NS \\
\hline Dyslipidemia $(n)$ & $5(28 \%)$ & $7(41 \%)$ & NS \\
\hline RAS inhibitors $(n)$ & & & NS \\
\hline ARB & $17(94 \%)$ & $17(100 \%)$ & \\
\hline ACEI & $1(6 \%)$ & $0(0 \%)$ & \\
\hline$\beta$ blocker $(n)$ & $5(28 \%)$ & $3(18 \%)$ & NS \\
\hline Statin $(n)$ & $4(22 \%)$ & $5(29 \%)$ & NS \\
\hline
\end{tabular}

Abbreviations: ACEI, angiotensin-converting enzyme inhibitor; ARB, angiotensin II type 1 receptor blocker; BP, blood pressure; RAS, renin-angiotensin system. 
a



b

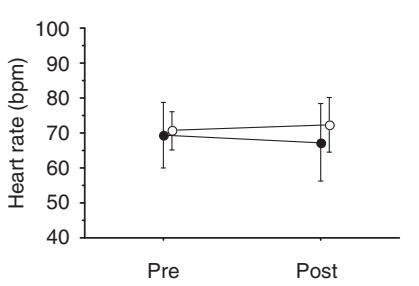

Figure 2 Changes in BP and heart rate during the study period. The BP and heart rate were almost the same between the cilnidipine (closed circles) and amlodipine (open circles) groups. Systolic and diastolic BP values were significantly reduced in both the groups after 6 months of treatment. Heart rate remained unchanged after 6 months of treatment in both the groups.

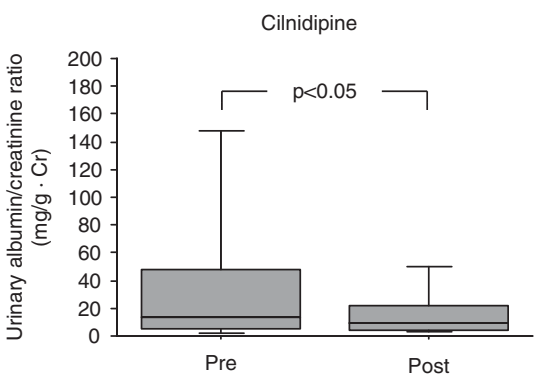

antihypertensive therapy compared with baseline values $(P<0.01)$. However, there were no significant differences in the BPs between the two groups (Figure 2a). There was no change in the heart rate after 6 months of treatment in either of the groups, although the heart rate tended to be decreased in the cilnidipine group (Figure $2 \mathrm{~b}$ ).

\section{Changes in urinary albumin}

The urinary albumin/creatinine ratio significantly decreased in the cilnidipine group after the 6-month treatment compared with that in the amlodipine group $(P<0.05$; Figure 3$)$.

\section{Changes in urinary 8-OHdG and L-FABP}

The urinary 8-OHdG (8-OHdG/creatinine ratio) and L-FABP levels (L-FABP/creatinine ratio) decreased significantly after 6-month cilnidipine treatment, whereas it did not change after amlodipine treatment (Figures 4 and 5, respectively). In addition, the urinary albumin, 8-OHdG and L-FABP reduction rates were not correlated with the systolic BP change rate (Figure 6).

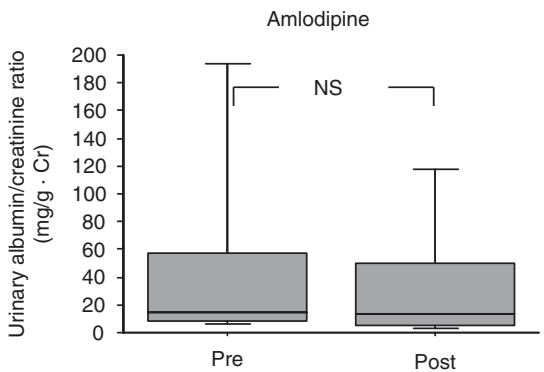

Figure 3 Changes in urinary albumin/creatinine ratio before and after the treatment. The urinary albumin/creatinine ratio was suppressed in the cilnidipine group but not in the amlodipine group.
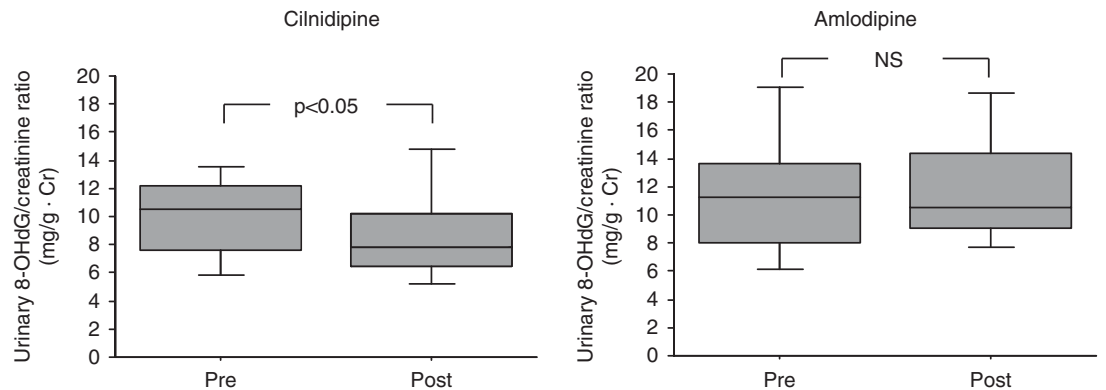

Figure 4 Changes in urinary $\mathrm{OHdG} /$ creatinine ratio before and after the treatment. The urinary $8-\mathrm{OHdG} / \mathrm{creatinine}$ ratio decreased significantly after treatment with cilnidipine but not after treatment with amlodipine.
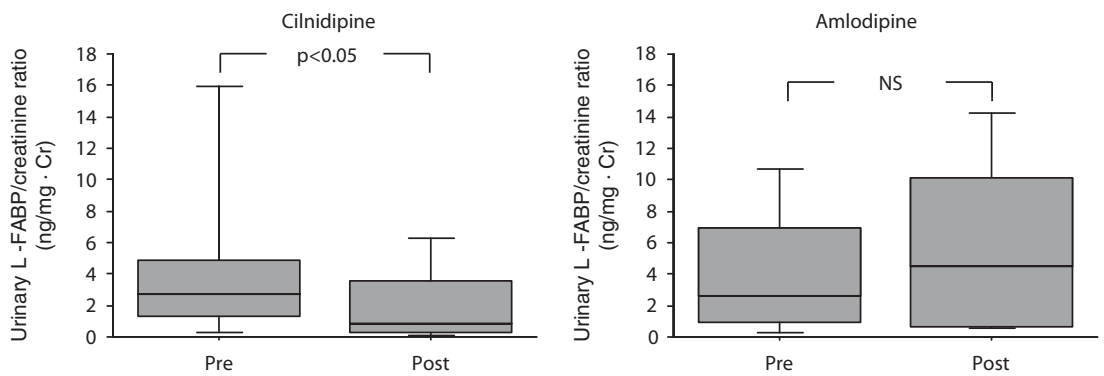

Figure 5 Changes in urinary L-FABP/creatinine ratio before and after the treatment. The urinary L-FABP/creatinine ratio decreased after treatment with cilnidipine but not after treatment with amlodipine. 

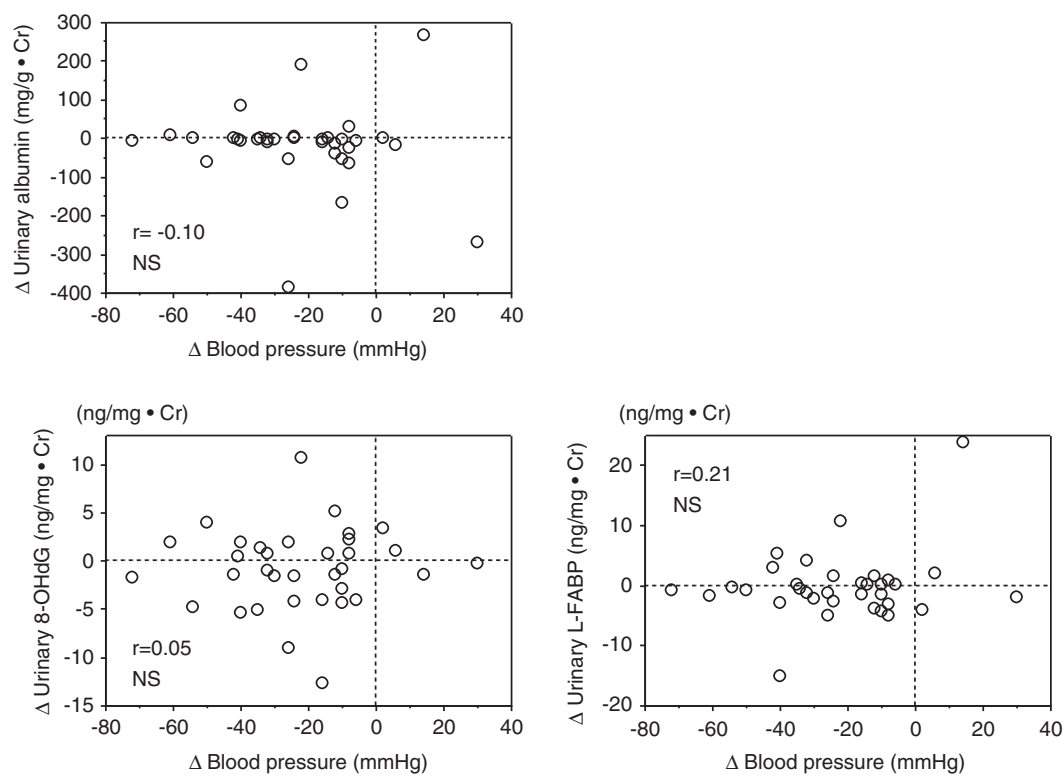

Figure 6 Correlation between change in BP and changes in urinary albumin, 8-OHdG and L-FABP before and after the treatment. No significant correlations were seen between the changes in urinary albumin/creatinine ratio, $8-\mathrm{OHdG} /$ creatinine ratio or L-FABP/creatinine ratio and the change in systolic BP before and after the treatment.

\section{DISCUSSION}

The results of the present study suggested that cilnidipine had greater antiproteinuric effects than amlodipine when used in combination with a RAS inhibitor to treat hypertensive patients. Furthermore, to our knowledge, this is the first study showing higher antioxidant activity of cilnidipine than amlodipine.

Our results are in agreement with those of previous studies, showing that cilnidipine had greater antiproteinuric effects than amlodipine when used in combination therapy. ${ }^{5,8}$ The Cilnidipine vs. Amlodipine Randomized Trial for Evaluation in Renal Disease study showed that cilnidipine was more beneficial than amlodipine in hypertensive patients. ${ }^{7}$

Besides RAS inhibition, strict control of BP is considered important for preventing the progression of kidney disease. ${ }^{15,17,18}$ Both cilnidipine and amlodipine effectively reduced BP level in hypertensive patients after 6 months of treatment. Furthermore, there was no significant correlation between change in BP and change in albuminuria level after a 6-month treatment with cilnidipine. These results suggest that the antiproteinuric effect of cilnidipine might be independent of the effect on BP, which is compatible with the results of the Cilnidipine vs. Amlodipine Randomized Trial for Evaluation in Renal Disease study.

The greater antiproteinuric effect and BP reduction accomplished by cilnidipine than amlodipine might be due to its N-type calciumchannel blockade action. Cilnidipine inhibits norepinephrine secretion ${ }^{19}$ and neurally stimulated renal vasoconstriction..$^{20}$ Because the sympathetic nerve activity affects both afferent and efferent arterioles, the inhibition of N-type calcium channels would dilate both the types of arterioles. Previous experimental studies have shown that cilnidipine causes substantial vasodilation of efferent and afferent arterioles in the canine kidney ${ }^{20}$ and that it reduces glomerular hypertension by causing the vasodilation of efferent arterioles. ${ }^{21} \mathrm{On}$ the other hand, only L-type CCBs are unable to reduce glomerular hypertension because the efferent arterioles lack L-type calcium channels. $^{22}$
Loss of redox homeostasis and formation of excessive free radicals have an important role in the pathogenesis of kidney disease and hypertension. The former contributes to the activation of the proinflammatory and profibrotic pathways in the kidney, leading to reduced vascular compliance and proteinuria. ${ }^{23}$ Dihydropyridine derivatives have been shown to act as lipophilic chain-breaking antioxidants. ${ }^{24} \mathrm{~A}$ recent study measured the ionomycin-stimulated superoxide production in cultured human mesangial cells and showed that cilnidipine has stronger antioxidant activity than amlodipine at the clinical plasma concentration. ${ }^{9}$ The stronger antioxidant activity of cilnidipine may be attributed to the greater difference in lipophilicity between cilnidipine and amlodipine, as reported by a previous study. ${ }^{25}$ Therefore, we hypothesized that cilnidipine might exert the renoprotective effect at least partially by suppressing oxidative stress that causes cellular injury and promotes glomerular and renal tubule injuries.

Another possibility for the cilnidipine-mediated suppression of proteinuria and oxidative stress in hypertensive patients receiving RAS-inhibitor treatment might be the inhibition of the renal RAS system by cilnidipine. Moreover, even previous studies reported better renoprotective effects of cilnidipine than amlodipine. ${ }^{26,27}$ Cilnidipine was shown to prevent the increase in renal angiotensin II content in spontaneously hypertensive rat/ND mcr-cp (an obese spontaneously hypertensive model). ${ }^{26}$ In addition, it reduced renal ACE activity and renal aldosterone content in uninephrectomized deoxycorticosterone-salt hypertensive rats. ${ }^{27}$ However, in the present study, the activity of renal RAS system in hypertensive patients was not evaluated.

Our study has several limitations. First, we could perform serial measurements of the urinary levels of albumin, 8-OHdG and L-FABP for only a limited number of patients and time points. Second, although we showed the collected data simultaneously for urinary albumin excretion, 8-OHdG and L-FABP, we could not show that antioxidant effects were the direct mechanism by which cilnidpine provided renoprotection in the present study. 
In conclusion, we showed that combination therapy with cilnidipine, and not amlodipine, ameliorated urinary albumin excretion in hypertensive patients receiving RAS-inhibitor treatment. Furthermore, cilnidipine decreased the urinary 8-OHdG and L-FABP levels in these patients. These data suggest that cilnidipine has greater renoprotective and antioxidative effects than amlodipine in hypertensive patients. Further research needs to be conducted to confirm the mechanisms underlying the beneficial effects of cilnidipine in hypertensive patients.

\section{CONFLICT OF INTEREST}

The authors declare no conflict of interest.

\section{ACKNOWLEDGEMENTS}

We thank Etsuko Uematsu for her assistance and contributions to the study. This study was supported in part by grants from the Ministry of Education, Culture, Sports, Science and Technology of Japan (Knowledge Cluster and Scientific Research on Innovative Areas) and the Ministry of Health, Labor and Welfare of Japan.

1 Kundhal K, Lok CE. Clinical epidemiology of cardiovascular disease in chronic kidney disease. Nephron Clin Pract 2005; 101: c47-c52.

2 Brenner BM, Cooper ME, de Zeeuw D, Keane WF, Mitch WE, Parving HH, Remuzzi G, Snapinn SM, Zhang Z, Shahinfar S. Effects of losartan on renal and cardiovascular outcomes in patients with type 2 diabetes and nephropathy. N Engl J Med 2001; 345: 861-869.

3 Wright Jr JT, Bakris G, Greene T, Agodoa LY, Appel LJ, Charleston J, Cheek D, DouglasBaltimore JG, Gassman J, Glassock R, Hebert L, Jamerson K, Lewis J, Phillips RA, Toto RD, Middleton JP, Rostand SG. Effect of blood pressure lowering and antihypertensive drug class on progression of hypertensive kidney disease: results from the AASK trial. JAMA 2002; 288: 2421-2431.

4 Rose GW, Kanno Y, Ikebukuro H, Kaneko M, Kaneko K, Kanno T, Ishida Y, Suzuki H. Cilnidipine is as effective as benazepril for control of blood pressure and proteinuria in hypertensive patients with benign nephrosclerosis. Hypertens Res 2001; 24 377-383.

5 Kojima S, Shida M, Yokoyama H. Comparison between cilnidipine and amlodipine besilate with respect to proteinuria in hypertensive patients with renal diseases. Hypertens Res 2004; 27: 379-385.

6 Lewis EJ, Hunsicker LG, Clarke WR, Berl T, Pohl MA, Lewis JB, Ritz E, Atkins RC, Rohde R, Raz I. Renoprotective effect of the angiotensin-receptor antagonist irbesartan in patients with nephropathy due to type 2 diabetes. N Engl J Med 2001; 345: 851-860.

7 Fujita T, Ando K, Nishimura H, Ideura T, Yasuda G, Isshiki M, Takahashi K. Cilnidipine versus Amlodipine Randomised Trial for Evaluation in Renal Desease (CARTER) Study investigators. Antiproteinuric effect of the calcium channel blocker cilnidipine added to renin-angiotensin inhibition in hypertensive patients with chronic renal disease. Kidney Int 2007; 72: 1543-1549.

8 Konda T, Enomoto A, Matsushita J, Takahara A, Moriyama T. The N- and L-type calcium channel blocker cilnidipine suppresses renal injury in Dahl rats fed a high-sucrose diet, an experimental model of metabolic syndrome. Nephron Physiol 2005; 101: 1-13.

9 Hishikawa K, Takase O, Idei M, Fujita T. Comparison of antioxidant activity of cilnidipine and amlodipine. Kidney Int 2009; 76: 230-231.

10 Valavanidis A, Vlachogianni T, Fiotakis C. 8-Hydroxy-2'-deoxyguanosine (8-OHdG): a critical biomarker of oxidative stress and carcinogenesis. J Environ Sci Health C Environ Carcinog Ecotoxicol Rev 2009; 27: 120-139.

11 Saito S, Yamauchi H, Hasui Y, Kurashige J, Ochi H, Yoshida K. Quantitative determination of urinary 8-hydroxyguanosine (8-OHdG) by using ELISA. Res Commun Mol Pathol Pharmacol 2000; 107: 39-44.
12 Nakamura T, Sato E, Fujiwara N, Kawagoe Y, Ueda Y, Suzuki T, Ueda S, Adachi H, Okuda S, Yamagishi S. Ezetimibe decreases serum levels of asymmetric dimethylarginine (ADMA) and ameliorates renal injury in non-diabetic chronic kidney disease patients in a cholesterol-independent manner. Pharmacol Res 2009; 60: 525-528.

13 Kamijo A, Sugaya T, Hikawa A, Yamanouchi M, Hirata Y, Ishimitsu T, Numabe A, Takagi M, Hayakawa H, Tabei F, Sugimoto T, Mise N, Kimura K. Clinical evaluation of urinary excretion of liver-type fatty acid-binding protein as a marker for the monitoring of chronic kidney disease: a multicenter trial. J Lab Clin Med 2005; 145: 125-133.

14 Tanaka T, Doi K, Maeda-Mamiya R, Negishi K, Portilla D, Sugaya T, Fujita T, Noiri E. Urinary L-type fatty acid-binding protein can reflect renal tubulointerstitial injury. $\mathrm{Am} \mathrm{J}$ Pathol 2009; 174: 1203-1211.

15 Ogihara T, Kikuchi K, Matsuoka H, Fujita T, Higaki J, Horiuchi M, Imai Y, Imaizumi T, Ito S, Iwao H, Kario K, Kawano Y, Kim-Mitsuyama S, Kimura G, Matsubara H, Matsuura H, Naruse M, Saito I, Shimada K, Shimamoto K, Suzuki H, Takishita S, Tanahashi N, Tsuchihashi T, Uchiyama M, Ueda S, Ueshima H, Umemura S, Ishimitsu T, Rakugi H. The Japanese Society of Hypertension guidelines for the management of hypertension (JSH 2009). Hypertens Res 2009; 32: 3-107.

16 Teramoto T, Sasaki J, Ueshima H, Egusa G, Kinoshita M, Shimamoto K, Daida H, Biro S, Hirobe K, Funahashi T, Yokote K, Yokode M, Japan Atherosclerosis Society (JAS) Committee for epidemiology and clinical management of atherosclerosis. Diagnostic criteria for dyslipidemia. Executive summary of Japan Atherosclerosis Society (JAS) guideline for diagnosis and prevention of atherosclerotic cardiovascular diseases for Japanese. J Atheroscler Thromb 2007; 14: 155-158.

17 Chobanian AV, Bakris GL, Black HR, Cushman WC, Green LA, Izzo Jr JL, Jones DW, Materson BJ, Oparil S, Wright Jr JT, Roccella EJ. The seventh report of the Joint National Committee on Prevention, Detection, Evaluation, and Treatment of High Blood Pressure: the JNC 7 report. JAMA 2003; 289: 2560-2572.

18 Mancia G, De Backer G, Dominiczak A, Cifkova R, Fagard R, Germano G, Grassi G, Heagerty AM, Kjeldsen SE, Laurent S, Narkiewicz K, Ruilope L, Rynkiewicz A, Schmieder RE, Boudier HA, Zanchetti A, Vahanian A, Camm J, De Caterina R, Dean V, Dickstein K, Filippatos G, Funck-Brentano C, Hellemans I, Kristensen SD, McGregor K, Sechtem U, Silber S, Tendera M, Widimsky P, Zamorano JL, Erdine S, Kiowski W, Agabiti-Rosei E, Ambrosioni E, Lindholm LH, Viigimaa M, Adamopoulos S, AgabitiRosei E, Ambrosioni E, Bertomeu V, Clement D, Erdine S, Farsang C, Gaita D, Lip G, Mallion JM, Manolis AJ, Nilsson PM, O'Brien E, Ponikowski P, Redon J, Ruschitzka F, Tamargo J, van Zwieten P, Waeber B, Williams B. 2007 Guidelines for the management of arterial hypertension: The task force for the management of arterial hypertension of the European Society of Hypertension (ESH) and of the European Society of Cardiology (ESC). J Hypertens 2007; 25: 1105-1187.

19 Nap A, Mathy MJ, Balt JC, Pfaffendorf M, van Zwieten PA. The evaluation of the N-type channel blocking properties of cilnidipine and other voltage-dependent calcium antagonists. Fundam Clin Pharmacol 2004; 18: 309-319.

20 Hayashi K, Wakino S, Sugano N, Ozawa Y, Homma K, Saruta T. Ca ${ }^{2}+$ channel subtypes and pharmacology in the kidney. Circ Res 2007; 100: 342-353.

21 Zhou $\mathrm{X}$, Ono $\mathrm{H}$, Ono Y, Frohlichzzz ED. N- and L-type calcium channel antagonist improves glomerular dynamics, reverses severe nephrosclerosis, and inhibits apoptosis and proliferation in an I-NAME/SHR model. J Hypertens 2002; 20: 993-1000.

22 Hansen PB, Jensen BL, Andreasen D, Skøtt O. Differential expression of T- and L-type voltage-dependent calcium channels in renal resistance vessels. Circ Res 2001; 89. 630-638.

23 Nistala R, Whaley-Connell A, Sowers JR. Redox control of renal function and hypertension. Antioxid Redox Signal 2008; 10: 2047-2089.

24 Mak IT, Weglicki WB. Comparative antioxidant activities of propranolol, nifedipine, verapamil, and diltiazem against sarcolemmal membrane lipid peroxidation. Circ Res 1990; 66: 1449-1452.

25 Uesawa Y, Mohri K. Relationship between lipophilicities of 1,4-dihydropyridine derivatives and pharmacokinetic interaction strengths with grapefruit juice. Yakugaku Zasshi 2008; 128: 117-122.

26 Fan YY, Kohno M, Nakano D, Ohsaki H, Kobori H, Suwarni D, Ohashi N, Hitomi H, Asanuma K, Noma T, Tomino Y, Fujita T, Nishiyama A. Cilnidipine suppresses podocyte injury and proteinuria in metabolic syndrome rats: possible involvement of $\mathrm{N}$-type calcium channel in podocyte. J Hypertens 2010; 28: 1034-1043.

27 Toba H, Yoshida M, Tojo C, Nakano A, Oshima Y, Kojima Y, Noda K, Wang J, Kobara M, Nakata T. L/N-type calcium channel blocker cilnidipine ameliorates proteinuria and inhibits the renal renin-angiotensin-aldosterone system in deoxycorticosterone acetatesalt hypertensive rats. Hypertens Res 2011; 34: 521-529. 